\title{
NON-PARALLEL PLANE RAYLEIGH BENARD CONVECTION IN CYLINDRICAL GEOMETRY
}

\author{
A. GOLBABAI (SHAYGAN)
}

\begin{abstract}
Summary. This paper considers the effect of a perturbed wall in regard to the classical Benard convection problem in which the lower rigid surface is of the form $z=\varepsilon^{2} g(s), s=\varepsilon r$, in axisymmetric cylindrical polar coordinates, $(r, \phi, z)$. The boundary conditions at $s=0$ for the linear amplitude equation is found and it is shown that these conditions are different from those which apply to the nonlinear problem investigated by Stewartson (1978) [2], representing a distribution of convection cells near the centre.
\end{abstract}

\section{Introduction}

The theoretical foundation of the on set of thermal instability in an infinite horizontal layer of fluid heated uniformly from below, was laid by Rayleigh (1916) [9], who proved the validity of the principle of the exchange of stabilities. For the case of two free boundaries, several papers recently have appeared in which the effect of certain perturbations of the classical Benard problem are studied. For example, Daniels (1978) [3], has studied the effect of including distant conducting side-walls at $x=O(L)$, when the Rayleigh number, $R$, exceeds the classical critical $R$ by $O\left(L^{-2}\right)$, where $L$ is large. Stewartson (1978) [2] has considered a similar problem but with distant cylindrical conducting boundary. And again

Received April 20, 1991.

This work was supported by the Iran University of Science and Technology while the author was on sabatical leave at Iowa State University of Science and Technology, Ames, Iowa. 
Daniels (1980) [4] has studied the effect of centrifugal force in a shallow rotating cylinder or annulus. These studies all result in certain changes in the amplitude equation, which results from the balance of terms in the governing equation at $O\left(\varepsilon^{3}\right)$.

The changes from the classical problem are essentially extra terms in the amplitude equation or changes in its boundary conditions. It is hoped that experimental results will be more easily compared with these modified problems.

In this paper we are interested in the Benard convection problem associated with the lower surface being of the form $z=\varepsilon^{2} g(s)$, in cylindrical geometry with axisymmetric. We refer to this new problem as the non parallel plane problem in contrast to the parallel plane problem, Golbabai (1986) [5], where the lower plane is given by $z=0$. The upper boundary is $z=1$. It is assumed that $g(s)$ is bounded so that for $\varepsilon$ sufficiently small the surfaces do not intersect. We choose $g(0)=0$ and $g$ positive for $r=\infty$, the excess of the Rayleigh number above $R_{c}$ (critical Rayleigh number) is assumed $O\left(\varepsilon^{2}\right)$, and the deviation of the lower surface from the planer case is $O\left(\varepsilon^{2}\right)$. We consider fluid confined between two rigid boundaries $z=1$ and $z=\varepsilon^{2} g(s)$ where $z, r$ are dimensionaless cylindrical coordinates and $\varepsilon$ is a small parameter. Gravity acts in the negative direction and the flow field extends to $r=0$ and $r=\infty$. For definiteness the space coordinates are made non-dimensional with respect to the fluid depth, $d$, the lower surface $z=\varepsilon^{2} g(s)$ is kept at constant temperature $\theta_{0}^{*}$ and the upper surface $z=1$ at a constant temperature $\theta_{1}^{*}$. The velocity component $v$ is taken to be zero.

\section{The governing equations of motion}

The full set of equations in the Oberbeck-Boussinesq approximation for viscous, incompressible, axisymmetric flow can be expressed as follows:

$$
\begin{aligned}
& u_{r}+\frac{u}{r}+w_{z}=0 \\
& u_{t}-\sigma\left(\nabla^{2} u-\frac{u}{r^{2}}\right)+p_{r}=-\left(u u_{r}+w u_{z}\right) \\
& w_{t}-\sigma\left(\nabla^{2} w+\theta\right)+p_{z}=-\left(u w_{r}+w w_{z}\right)
\end{aligned}
$$




$$
\begin{aligned}
& \theta_{t}-\nabla^{2} \theta-R w=-\left(u \theta_{r}+w \theta_{z}\right) \\
& \nabla^{2}=\frac{\partial^{2}}{\partial r^{2}}+\frac{1}{r} \frac{\partial}{\partial r}+\frac{\partial^{2}}{\partial z^{2}}
\end{aligned}
$$

where

$$
u_{r}=\frac{\partial u}{\partial r}, \quad u_{z}=\frac{\partial u}{\partial z}, \ldots
$$

and

$$
R=g \alpha_{0} d^{3}\left(\theta_{0}^{*}-\theta_{1}^{*}\right) / \kappa \nu, \quad \sigma=\frac{\nu}{\kappa},
$$

is the Rayleigh number and Prandtl number respectively.

We define the slow variable, $s$, by $s=\varepsilon r$, where $\varepsilon$ is a small parameter. The boundary conditions are

$$
u=w=\theta=0 \text { at } z=1, \varepsilon^{2} g(s) .
$$

3. Analysis of the base flow and-steady state solution

For steady flow in the parallel plane problem, which is given by $\varepsilon=0$, there is a solution of the form:

$$
\begin{aligned}
& u=0, \quad w=0 \\
& \theta=0, \quad p=\text { constant }
\end{aligned}
$$

for $\varepsilon \neq 0$ we denote the velocity component of the steady case flow by $u_{s}, w_{s}$ and pressure, temperature by $p_{s}, \theta_{s}$ respectively. The boundary conditions for the base flow are

$$
\begin{aligned}
& u_{s}=w_{s}=\theta_{s}=0 \quad \text { at } \quad z=1 \\
& u_{s}=w_{s}=0, \theta_{s}=R \varepsilon^{2} g(s) \text { at } \quad z=\varepsilon^{2} g(s) .
\end{aligned}
$$

We also add the condition that

$$
u_{s} \rightarrow 0 \text { and } w_{s} \rightarrow 0 \text { as } s \rightarrow \infty
$$


For small $\varepsilon$ we expand the perturbations $u_{s}, w_{s}, \theta_{s}$ and $p_{s}$ in powers of $\varepsilon$ and write:

$$
\begin{aligned}
u_{s} & =\varepsilon u_{1}+\varepsilon^{2} u_{2}+\ldots \\
w_{s} & =\varepsilon w_{1}+\varepsilon^{2} w_{2}+\ldots \\
\theta_{s} & =\varepsilon \theta_{1}+\varepsilon^{2} \theta_{2}+\ldots \\
p_{s} & =\varepsilon p_{1}+\varepsilon^{2} p_{2}+\ldots
\end{aligned}
$$

The functions $u_{i}, w_{i}, \theta_{i}, p_{i}$, for $i=1,2, \ldots$ are considered to depend on the two variables $z$ and $s$.

Substituting the form of expansions (3.1)-(3.3) into (2.1)-(2.4), replacing $\frac{\partial}{\partial r}$ by $\varepsilon \frac{\partial}{\partial s}$, and equating powers of $\varepsilon$, we obtain a set of partial differential equations as follows:

From (2.1), we find that,

$$
\frac{\partial w_{1}}{\partial z}=0, \quad u_{i}+s\left(\frac{\partial u_{i}}{\partial s}+\frac{\partial w_{i+1}}{\partial z}\right)=0, \quad i=1,2,3
$$

from (2.2) and (2.3),

$$
\begin{aligned}
& \frac{\partial^{2} u_{1}}{\partial z^{2}}=0, \quad \sigma \frac{\partial^{2} u_{i+1}}{\partial z^{2}}-\frac{\partial p_{i}}{\partial s}=0, \quad i=1,2 \\
& \frac{\partial p_{1}}{\partial z}-\sigma \theta_{1}=0, \frac{\partial p_{i}}{\partial z}-\sigma\left(\theta_{i}+\frac{\partial^{2} w_{i}}{\partial z^{2}}\right)=0, i=2,3
\end{aligned}
$$

Finally from (2.4),

$$
\begin{gathered}
\theta_{1}=0, \quad \frac{\partial^{2} \theta_{i}}{\partial z^{2}}+R w_{i}=0, \quad i=2,3 \\
\frac{1}{s} \frac{\partial \theta_{2}}{\partial s}+\frac{\partial^{2} \theta_{4}}{\partial z^{2}}+R w_{4}-w_{2} \frac{\partial \theta_{2}}{\partial z}=0 .
\end{gathered}
$$

Now we define the boundary conditions on $u_{i}, \theta_{i}, w_{i}, i=1,2, \ldots$ From (3.1),

$$
u_{i}=w_{i}=\theta_{i}=0 \text { at } z=1, i=1,2,3, \ldots
$$


The boundary conditions on $z=\varepsilon^{2} g$, for $u_{i}, w_{i}, \theta_{i}(i=1,2, \ldots)$, are given by expansion of $u_{s}, w_{s}, \theta_{s}$ about $z=0$, and the details of this work are given for $u_{s}$ only; we have

$$
\begin{aligned}
u_{s}\left(s, \varepsilon^{2} g\right)=\varepsilon u_{1}(s, 0) & +\varepsilon^{2} u_{2}(s, 0)+\varepsilon^{3}\left(g u_{1 z}+u_{3}\right)_{z=0} \\
& +\varepsilon^{4}\left(g u_{2 z}+u_{4}\right)_{z=0}+\ldots
\end{aligned}
$$

Therefore, since $u_{s}\left(s, \varepsilon^{2} g\right)=0$,

$$
u_{1}=u_{2}=0, \quad g u_{1 z}+u_{3}=g u_{2 z}+u_{4}=0 \text { on } z=0
$$

where

$$
u_{2 z}=\frac{\partial u_{i}}{\partial z} \quad(i=1,2,4) .
$$

Similar arguments provide boundary conditions for $w_{i}$ and $\theta_{i}$ on $z=0$.

The solutions of the equations (3.4)-(3.8) subject to the boundary condtions are listed below.

$$
\begin{aligned}
& u_{1}=u_{2}=0, \quad w_{1}=w_{2}=w_{3}=0, \quad \theta_{1}=\theta_{3}=p_{1}=0 \\
& \theta_{2}=R(1-z) g \\
& p_{2}=\sigma R\left(z-\frac{z^{2}}{2}\right)+B(s) \\
& u_{3}=R\left(z^{3}-\frac{z^{4}}{24}-\frac{z}{8}\right) \frac{d g}{d s}+\sigma^{-1}\left(z^{2}-z\right) \frac{d B}{d s} / 2 \\
& w_{4}=R f_{1}(z)\left(\frac{d^{2} g}{d s^{2}}+\frac{1}{s} \frac{d g}{d s}\right)+\sigma^{-1} f_{2}(z)\left(\frac{d^{2} B}{d s^{2}}+\frac{1}{s} \frac{d B}{d s}\right)
\end{aligned}
$$

where $f_{1}$ and $f_{2}$ are given by

$$
\begin{aligned}
& f_{1}=\frac{z^{5}}{120}+\frac{{ }^{\circ} z^{2}}{16}-\frac{z^{4}}{24} \\
& f_{2}=\frac{z^{2}}{2}-\frac{z^{3}}{3} .
\end{aligned}
$$

In obtaining these solution, $p_{2}$ was first found in the form (3.13), where $B(s)$ is an unknown function at this stage. 
In order that $u_{3} \rightarrow 0$ as $s \rightarrow \infty$, we see from (3.14) that

$$
\frac{d g}{d s} \rightarrow 0 \text { as } s \rightarrow \infty
$$

and

$$
\frac{d B}{d s} \rightarrow 0 \text { as } s \rightarrow \infty
$$

From the condition $w_{4}=0$ on $z=1$, and (3.17) we find that

$$
\frac{d B}{d s}+(7 \sigma R / 20) \frac{d g}{d s}=0
$$

and thus

$$
B=(-7 \sigma R / 20) g(s)+\text { const. }
$$

Substituting (3.20) into (3.13)-(3.15), provides the explicit form of $p_{2}, u_{3}, w_{4}$. Summarizing, the expansions for the base flow, pressure and temperature can be written as follows:

$$
\begin{aligned}
& u_{s}=\varepsilon^{3} \mathbb{R}\left(1-\frac{z^{4}}{24}+\frac{z^{3}}{16}-7 \frac{z^{2}}{40}+\frac{z}{20}\right) \frac{d g}{d s}+\ldots \\
& w_{s}=\varepsilon^{4}\left(z^{5}-5 z^{4}+7 z^{3}-3 z^{3}\right)\left(\frac{d^{2} g}{d s^{2}}+\frac{1}{s} \frac{d g}{d s}\right) \mathbb{R} / 120+\ldots \\
& \theta_{s}=\varepsilon^{2} R(1-z) g+\ldots \\
& p_{s}=\varepsilon^{2} \sigma\left(z-\frac{z^{2}}{2}-\frac{7}{20}\right) g+\ldots
\end{aligned}
$$

It is noted that as $s \rightarrow \infty$, we have zero fluid velocity and just a linear temperature variation.

\section{The disturbance equations in matrix form}

We continue with the case where the equation of the lower boundary is $z=\varepsilon^{2} g(s)$, where $s=\varepsilon r$. In equations (2.1)-(2.4) we set

$$
\begin{aligned}
u & =u_{s}+\hat{u}(r, z, t), \\
w & =w_{s}+\hat{w}(r, z, t), \\
\theta & =\theta_{s}+\hat{\theta}(r, z, t), \\
p & =p_{s}-\hat{p}(r, z, t),
\end{aligned}
$$


(where the minus sign with perturbed pressure is merely for convenience) to obtain the equations for small disturbances $\hat{u}, \hat{w}, \hat{\theta}, \hat{p}$, these are assumed to be sufficiently small for nonlinear products of these terms to be neglected in the governing equations. The functions $u_{s}, w_{s}, \theta_{s}, p_{s}$ are the steady solutions of (2.1)-(2.4) which are given in (3.21)-(3.24). Upon substitution of (4.1) into (2.1)-(2.4) we then obtain the linear system

$$
\begin{aligned}
& \frac{\partial \hat{u}}{\partial r}+\frac{\hat{u}}{r}+\frac{\partial \hat{w}}{\partial z}=0, \\
& \frac{\partial \hat{u}}{\partial t}+u_{s} \frac{\partial \hat{u}}{\partial r}+\hat{u} \frac{\partial u_{s}}{\partial r}+\hat{w} \frac{\partial u_{s}}{\partial z}+w_{s} \frac{\partial \hat{u}}{\partial z}=\hat{p}_{r}+\sigma\left(\nabla^{2} \hat{u}-\frac{\hat{u}}{r^{2}}\right) \\
& \frac{\partial \hat{w}}{\partial t}+u_{s} \frac{\partial \hat{w}}{\partial r}+\hat{u} \frac{\partial w_{s}}{\partial r}+\hat{w} \frac{\partial w_{s}}{\partial z}+w_{s} \frac{\partial \hat{w}}{\partial z}=\hat{p}_{z}+\sigma\left(\nabla^{2} \hat{w}+\hat{\theta}\right) \\
& \frac{\partial \hat{\theta}}{\partial t}+u_{s} \frac{\partial \hat{\theta}}{\partial r}+\hat{u} \frac{\partial \theta_{s}}{\partial r}+\hat{w} \frac{\partial \theta_{s}}{\partial z}+w_{s} \frac{\partial \hat{\theta}}{\partial z}=R \hat{w}+\nabla^{2} \hat{\theta}
\end{aligned}
$$

and

$$
\begin{aligned}
& u=u_{s}+\tilde{u}(r, t) \\
& \hat{u}=\hat{\theta}=\hat{w}=0 \text { at } z=1, \quad \varepsilon^{2} g(s) .
\end{aligned}
$$

We now introduce the notation

$$
\tilde{u}=\frac{\partial \hat{u}}{\partial z}, \quad \tilde{\theta}=\frac{\partial \hat{\theta}}{\partial z}
$$

and in equation (4.3) we write,

$$
\nabla^{2} \hat{u}=\frac{\partial^{2} \hat{u}}{\partial r^{2}}+\frac{1}{r} \frac{\partial \hat{u}}{\partial r}+\frac{\partial \tilde{u}}{\partial z} .
$$

The (4.3) expresses $\frac{\partial \tilde{u}}{\partial z}$ in terms of $\hat{u}, \tilde{u}, \hat{w}, \hat{p}$ and their derivatives with respect to $r$ and $t$. We next note that the derivative with respect to $z$ of (4.2) may be written as

$$
\frac{\partial^{2} \hat{w}}{\partial z^{2}}=-\left(\frac{\tilde{u}}{r}+\frac{\partial \tilde{u}}{\partial r}\right)
$$

In our analysis we shall ignor powers of $\varepsilon^{n}$ for $n \geq 3$. With this assumption and (3.21)-(3.24) we observe that

$$
u_{s}=0, \quad w_{s}=0, \quad \theta_{s}=\varepsilon^{2} \operatorname{Rg}(1-z)
$$


Now we introduce the extended flow vector

$$
\underline{U}=\left[\tilde{u}, \frac{\partial \hat{p}}{\partial r}, \frac{\partial \tilde{\theta}}{\partial r}, \hat{u}, \frac{\partial \hat{w}}{\partial r}, \frac{\partial \hat{\theta}}{\partial r}\right]^{t r}
$$

where $t r$ denotes the transpose and $\tilde{u}, \tilde{\theta}$ are given in (4.7). Substituting (4.10) in (4.3)-(4.5), we find that

$$
\left[\begin{array}{l}
\frac{\partial \hat{u}}{\partial z}=\sigma^{-1} \frac{\partial \hat{p}}{\partial r}-L \hat{u}+\sigma^{-1} \frac{\partial \hat{u}}{\partial t} \\
\frac{\partial^{2} \hat{p}}{\partial z \partial r}=\sigma\left(L\left(\tilde{u}+\frac{\partial \hat{w}}{\partial r}\right)+\frac{\partial \hat{\theta}}{\partial r}+\frac{\partial^{2} \hat{w}}{\partial r \partial t}\right) \\
\frac{\partial^{2} \hat{\theta}}{\partial z \partial r}=-R \frac{\partial \hat{w}}{\partial r}-L\left(\frac{\partial \hat{\theta}}{\partial r}\right)+\frac{\partial^{2} \hat{\theta}}{\partial r \partial t}+\frac{\partial \hat{w}}{\partial r} \times \frac{\partial \theta_{s}}{\partial z} \\
\frac{\partial \hat{u}}{\partial z}=\tilde{u} \\
\frac{\partial^{2} \hat{w}}{\partial z \partial r}=-L \hat{u} \\
\frac{\partial^{2} \hat{\theta}}{\partial r \partial z}=\frac{\partial \tilde{\theta}}{\partial r}
\end{array}\right.
$$

where $L$ is a linear operator: $L=\frac{\partial^{2}}{\partial r^{2}}+\frac{1}{r} \frac{\partial}{\partial r}-\frac{1}{r^{2}}$. This formulation enables us to write the equation (4.12) in matrix form:

$$
\frac{\partial \underline{U}}{\partial z}=A \underline{U}+B \frac{\partial \underline{U}}{\partial t}
$$

where $A$ and $B$ are matrices of order $(6,6)$.

$$
\begin{aligned}
A & =\left(\begin{array}{cccccc}
0 & 1 & 0 & -L & 0 & 0 \\
L & 0 & 0 & 0 & L & 1 \\
0 & 0 & 0 & 0 & X & -L \\
1 & 0 & 0 & 0 & 0 & 0 \\
0 & 0 & 0 & -L & 0 & 0 \\
0 & 0 & 1 & 0 & 0 & 0
\end{array}\right) \text { and } X=\left(1+\varepsilon^{2} g\right) R \\
B & =\left(\begin{array}{cccc} 
& \sigma^{-1} & 0 & 0 \\
0 & 0 & \sigma^{-1} & 0 \\
0 & 0 & 0 & 1
\end{array}\right)
\end{aligned}
$$

where $\underline{0}$ is the zero matrix of order $(3,3)$, we can write matrix $A$ in the form $A=A_{1}-L A_{2}+R\left(1+\varepsilon^{2} g\right) A_{3}$, where $A_{1}, A_{2}, A_{3}$ are constant matrix of order $(6,6)$. From (4.6)

$$
\hat{u}=\frac{\partial \hat{w}}{\partial r}=\frac{\partial \hat{\theta}}{\partial r}=0 \quad \text { at } \quad z=1
$$


On $z=\varepsilon^{2} g$, we have, for example, $\frac{\partial \hat{w}}{\partial r}=0$, so that

$$
\frac{\partial \hat{w}}{\partial r}=\frac{\partial \hat{w}(r, 0)}{\partial r}+\varepsilon^{2} g \frac{\partial^{2} w(r, 0)}{\partial z \partial r}+\ldots
$$

and so the order of approximation considered here, $\frac{\partial \hat{w}}{\partial r}=0$ at $z=0$. The complete set of boundary conditions can be conveniently labeled as follows:

$\gamma$ : The last three components of $\underline{U}$ are zero at $z=0$ and $z=1$ or alternatively:

$$
\gamma: C \underline{U}=0 \quad \text { at } \quad z=0, z=1
$$

where $C=\left(\begin{array}{cc}\underline{0} & \underline{0} \\ \underline{0} & I_{3}\end{array}\right)$, and $I_{3}$ is a unit matrix of order (3.3).

\section{Expansion procedure and derivation of amplitude equation}

If $g(s)=0$ (or $\varepsilon=0$ ) then we have the standard linear parallel plane problem, so that we may expect that the critical disturbance of plane problem will have a corresponding perturbed solution in the non parallel case. We assume $g(s)$ remains $O(1)$ for $r=O\left(\varepsilon^{-1}\right)$, and look for a steady solution of (4.13) in this region of the form

$$
\underline{U}=e^{i \alpha r} \underline{V}(z, s)+c \cdot c
$$

with $R=R_{c}+\varepsilon^{2} \beta$, where $\varepsilon$ is fixed by the size of the depression in the lower surface $\left(z=\varepsilon^{2} g\right)$, and $\beta$ is an arbitrary parameter which represent an $O\left(\varepsilon^{2}\right)$ variation in $R$ about $R_{c}$, and the symbol $c \cdot c$ denotes the complex conjugate. Now we expand the complex functions $\underline{V}$ in power or $\varepsilon$ :

$$
\underline{V}=\varepsilon \underline{F}_{1}+\varepsilon^{2} \underline{F}_{2}+\varepsilon^{3} \underline{F}_{3}+\ldots
$$

where $F_{i}$ is a function of $s$ and $z$. On substituting (5.1)-(5.2) into (4.13) and equating powers of $\varepsilon^{n}$, we obtain a set of equations as follows:

$$
\begin{aligned}
& L_{0} \underline{F}_{1}=0, \quad \gamma, \\
& L_{0} \underline{F}_{2}=-i \alpha_{c} A_{2} L_{1} \underline{F}_{1}, \quad \gamma, \\
& L_{0} \underline{F}_{3}=\left(R_{c} g+\beta\right) A_{3} \underline{F}_{1}-i \alpha A_{2} L_{1} \underline{F}_{2}-A_{2} L_{2} \underline{F}_{1}
\end{aligned}
$$


with

$$
C\left(\underline{F}_{3}-g \underline{F}_{1}\right)=0 \text { at } z=0, C \underline{F}_{3}=0 \text { at } z=1
$$

where

$$
\begin{aligned}
& L_{0}=\frac{\partial}{\partial z}-\left(A_{1}+\alpha^{2} A_{2}+R_{c} A_{3}\right) \\
& L_{1}=\frac{\partial}{\partial s}+\frac{1}{2 s} \\
& L_{2}=\frac{\partial^{2}}{\partial s^{2}}+\frac{1}{s} \frac{\partial}{\partial s}-\frac{1}{s^{2}},
\end{aligned}
$$

and $A_{1}, A_{2}, A_{3}$ are constant matrices of order $(6,6)$, also matrix $C$ and $\gamma$ are given in (4.16) and $\alpha, R_{c}$ are critical wave and Rayleigh number respectively.

A general solution (5.3) can be written as

$$
\underline{F}_{1}=\bar{A}(s) \underline{f}_{1}(z)
$$

where

$$
L_{0} \underline{f}_{1}(z)=0, \quad \gamma,
$$

and $f_{1}(z)$ is the critical eigenfunction of the standard parallel plane problem, S. Chandrasekhar 1961 [1]. The solution given in (5.7) contains an amplitude function, $\bar{A}(s)$, which is determined by solvability condition obtained at higher order $O\left(\varepsilon^{3}\right)$. Using (5.7), equation (5.4) becomes

$$
L_{0} \underline{F}_{2}=-i \alpha A_{2} L_{1} \bar{A}(s) \underline{f}_{1}: \gamma
$$

and has a solution for $\underline{F}_{2}$, if the adjoint condition:

$$
i \alpha L_{1} \bar{A}(s) \int_{0}^{1} \underline{f}(z)\left(A_{2} \underline{f}_{1}\right) d z=0
$$

is satisfied, the adjoint condition here is similar to that of Eagles 1980 [6], where $\underline{f}(z)$ is adjoint function.

From the boundary conditions on $\underline{F}_{2},(5.4)$, and the right-hand side $\underline{F}_{2}$ may be expressed as

$$
\underline{F}_{2}=-i\left(\frac{d \bar{A}}{d s}+\frac{\bar{A}}{2 s}\right) \underline{f}_{2}+\bar{A}_{1}(s) \underline{f}_{1}
$$


where $\bar{A}_{1}(s)$ is unknown function at this stage, and

$$
L_{0} \underline{f}_{2}=2 \alpha A_{2} \underline{f}_{1}: \gamma \text {. }
$$

A solution for $\underline{F}_{3}$ exists if the adjoint condition is satisfied, which results the amplitude equation.

$$
a\left(\frac{d^{2} \bar{A}}{d s^{2}}+\frac{1}{s} \frac{d \bar{A}}{d s}-\frac{\bar{A}}{4 s^{2}}\right)+\bar{A}\left\{\left(b-c R_{c}\right) g+b \beta\right\}=0 .
$$

Here

$$
\begin{aligned}
a & =-\alpha \int_{0}^{1} \underline{f}(z)\left(A_{2} \underline{f}_{2}\right) d z, \\
b & =\int_{0}^{1} \underline{f}(z)\left(A_{3} \underline{f}_{1}\right) d z, \\
c & =\sum_{k=4}^{6} f_{k} \frac{d f_{1 k}}{d z} \quad \text { at } \quad z=0
\end{aligned}
$$

where $f_{k}(z)$ is the $k$-th component of the adjoint function $\mathrm{f}(z)$, and $f_{1 k}(z)$ is the $k$-th component of eigenfunction $\mathrm{f}_{1}(z)$ and $A_{2}, A_{3}$ are given in (5.6). Now let $A_{0}(s)=\bar{A} s^{1 / 2}$, from $(5.10)$, we see that

$$
\frac{d^{2} A_{0}}{d s^{2}}+\left(\delta_{1}+\delta_{2} g\right) A_{0}=0
$$

where $\delta_{1}=\frac{b \beta}{a}, \delta_{2}=\frac{b-c R_{c}}{a}$.

The equation (5.11) is similar to that obtained by Eagles (1980) [6] in the two dimensional case where the boundary conditions are defined at $s= \pm \infty$. In our case, we have no information about the boundary condition at the center, $s=0$, and we shall investigate this condition by using a matching procedure.

\section{The inner solution and investigating of the amplitude equation}

In the neighborhood of $r=0$ the function $g(s)$ tends to zero and we look for a linearized solution of (2.1)-(2.4), in which $R=R_{c}$ and the components of the disturbance are given in terms of Bessel functions. An equivalent solution has been found in the stress-free case by Stewartson (1978) [2]. One solution is 


$$
\theta=h(z) J_{0}(\alpha r), u=f(z) J_{0}^{\prime}(\alpha r), w=g(z) J_{0}(\alpha r)
$$

and a second solution can be found by writing

$$
\begin{aligned}
u & =\bar{f}(z) J_{0}^{\prime}(\alpha r)+r f(z) J_{0}^{\prime \prime}(\alpha r) \\
w & =\bar{g}(z) J_{0}(\alpha r)+r g(z) J_{0}^{\prime}(\alpha r) \\
\theta & =\bar{h}(z) J_{0}(\alpha r)+r h(z) J_{0}^{\prime}(\alpha r)
\end{aligned}
$$

where the numerical solutions of $f, g, h, \bar{f}, \bar{g}, \bar{h}$ can be found.

The general solution for $\theta$ in the inner zone may now be written as

$$
\theta_{I}=\lambda J_{0}(\alpha r) h+\mu\left\{J_{0}(\alpha r) \bar{h}+r J_{0}^{\prime}(\alpha r) h\right\}
$$

$\theta_{I}$ denotes the inner solution and we use $\theta_{O}$ to denote the solution already found. In order to match (6.2) with the outer solution (5.1), we need the behavior of the amplitude function $A_{0}(s)$ as $s \rightarrow 0$, which is found to the

$$
A_{0} \sim \bar{a}+\bar{b} s+\ldots \quad(s \rightarrow 0)
$$

where $\bar{a}, \bar{b}$ are arbitrary constant and $s=\varepsilon r$.

From $(5.1),(5.7),(5.11)$ in the outer region where $g(s) \neq 0, \theta_{O}$ is given by

$$
\theta_{O} \sim \frac{e^{i \alpha r}}{s^{1 / 2}}\left\{h A_{0}-i \varepsilon \frac{\partial A_{0}}{\partial s} \bar{h}+\ldots\right\}+c \cdot c .
$$

Now the asymptotic expansion of (6.2) for large $r$ is:

$$
\theta_{I} \sim\left(\frac{2}{\pi \alpha r}\right)^{1 / 2}\left\{\left(\lambda-\frac{3 \mu}{8 \alpha}\right) h \cos \hat{r}+\mu \bar{h} \cos \hat{r}-\mu r h \sin \hat{r}\right\}
$$

where $\hat{r}=\alpha r-\frac{\pi}{4}$. Substituting (6.3) into (6.4) we obtain

$$
\theta_{O} \sim \frac{e^{i \alpha r}}{s^{1 / 2}}\{\bar{a} h+\varepsilon \bar{b} r h-i \bar{b} \varepsilon \bar{h}\}+c \cdot c
$$


and comparing (6.5) with (6.6), we see that a match of the terms in $h$ and $r h$ is secured if, respectively

$$
\begin{aligned}
& \bar{a}=\left(\frac{\varepsilon}{2 \pi \alpha}\right)^{1 / 2}\left(\lambda-\frac{3 \mu}{8 \alpha}\right) e^{-i \pi / 4} \\
& \varepsilon^{1 / 2} \bar{b}-\left(\frac{\pi \alpha}{2}\right)^{-1 / 2} e^{i \pi / 4}=0
\end{aligned}
$$

Therefore, from matching conditions (6.7) we can list the boundary conditions for the amplitude equation as:

$$
\begin{aligned}
& A_{0}(0)=b_{1} e^{-i \pi / 4} \\
& A_{0}^{\prime}(0)=b_{2} e^{i \pi / 4}
\end{aligned}
$$

where $b_{1}$ and $b_{2}$ are real constants. Note that these condtions are quite different from those which apply to the non-linear problem studied by Brown and Stewartson (1978) [2].

Now suppose that $g(s) \neq 0$, and define, $g_{1}(s)=-\delta_{2} g(s)$, so that the amplitude equation is given by, $A_{0}^{\prime \prime}+\left(\delta_{1}-g_{1}(s)\right) A_{0}=0$, where $g_{1}(s) \geq 0$, in view of the conditions (6.8), we set

$$
A_{0}=e^{-i \pi / 4}\left(\bar{A}_{1}(s)+i \bar{A}_{2}(s)\right)
$$

where $\bar{A}_{1}(s)$ and $\bar{A}_{2}(s)$ are assumed real functions, then

$$
\begin{array}{ll}
\bar{A}_{1}(0)=b_{1}, & \bar{A}_{2}(0)=0, \\
\bar{A}_{1}^{\prime}(0)=0, & \bar{A}_{2}^{\prime}(0)=B_{2}
\end{array}
$$

Let $\tilde{A}=\bar{A}_{1}+i \bar{A}_{2}$ and $\tilde{A}=\bar{R}(s) e^{i \psi(s)}$, then $\bar{R}$ and $\psi$ satisfy the equations:

$$
\begin{gathered}
\bar{R}^{\prime \prime}+\left(\delta_{1}-g_{1}\right) \bar{R}-(\psi)^{2} \bar{R}=0 \\
2 \psi^{\prime} \bar{R}^{\prime}+\psi^{\prime \prime} \bar{R}=0
\end{gathered}
$$

with boundary conditions

$$
\begin{array}{ll}
\bar{R}(0)=b_{1}, & \psi(0)=0 \\
\bar{R}^{\prime}(0)=0, & \psi^{\prime}(0)=\frac{b_{2}}{b_{1}}
\end{array}
$$


from $(6.11), \psi^{\prime}=\frac{c}{(\bar{R})^{2}}$ where $c$ is an arbitrary constant, and the boundary conditions at $s=0$ imply that, $c=b_{1} b_{2}$. Hence

$$
\psi=b_{1} b_{2} \int \frac{d s}{(\bar{R})^{2}} .
$$

Now if we impose the condition that $\bar{R} \rightarrow 0(s \rightarrow \infty)$, then we choose $c=0$, so that

$$
\begin{aligned}
& \bar{R}^{\prime \prime}+\left(\delta_{1}-g_{1}\right) \bar{R}=0 \\
& \bar{R}^{\prime}(0)=0 \\
& \vec{R} \rightarrow 0 \quad(s \rightarrow \infty)
\end{aligned}
$$

This system is the same as the linear form considered by Eagles (1980), [6], where the function $g_{1}(s)$ is taken to be $g_{1}(s)=\left(\tanh \frac{s}{\sqrt{2}}\right)^{2}$ and for $\delta_{1}=1 / 2$ there is a solution $\bar{R}=b_{1} \sec h \frac{s}{\sqrt{2}}$, in which $\bar{R} \rightarrow 0$ as $s \rightarrow \infty$, representing a distribution of convection cells concentrated near the center.

\section{Discussion}

It is well known that the base flow in the parallel plane problem $(g(s)=$ 0 , or $\varepsilon=0$ ), is unstable for $R>R_{c}$ and that for $R>R_{c}$ a pattern of convection cells or rolls is set up. In the non-parallel plan case we see that the local Rayleigh number is larger near $s=0$ than at $s=\infty$, so that the convection cells occur in the center more readily than away from the center and the positive value of $g(s)$ for $s>0$, causes an effective increase in the critical Rayleigh number over that for the plane case where $g(s)=0$. Finally, it should be pointed out that the boundary conditions at $s=0$ for the linear amplitude equation differs from those which apply to the non-linear problem investigated by Brown and Stewarson [2].

\section{References}

[1] S. Chandrasekhar, "Hydrodynamic and Hydromagnetic Stability Theory", Oxford University Press, London, 1961.

[2] S. N. Brown and K. Stewartson, Proc. R. Soc. London, A 360 (1978), 455.

[3] P. G. Daniels, Proc. R. Soc. London, A 363 (1978), 455. 
[4] P. G. Daniels, J. Fluid Mech., 99 (1980), 65.

[5] A. Golbabai, J. of Computational and Applied Mathematics, 16 (1986), 355.

[6] P. G. Eagles, J. Fluid Mech., 95 (1980), 166.

[7] E. L. Koschmieder, Beiter. Phys. Atmos., 39 (1966), 1.

[8] G. Veronis, J. Fluid Mech., 5 (1959), 401.

[9] Rayleigh, Lord, Phil. Mag., 32 (1916), 529.

Mathematics Department, Iowa State University of Science and Technology, Ames, Iowa 50011 U.S.A. 